\title{
On Flatness-based Control for Shape-Memory Alloy Actuators *
}

\author{
L. Kiltz* J. Rudolph ${ }^{* *}$ D. Gerbet ${ }^{* *}$ \\ * Corporate Research and Development, ZF Friedrichshafen AG \\ (e-mail: lothar.kiltz@zf.com) \\ ** Chair of Systems Theory and Control Engineering, Saarland \\ University (e-mail: \{j.rudolph,d.gerbet\}@lsr.uni-saarland.de)
}

Keywords: Flatness-based control, Shape-memory alloys

\section{INTRODUCTION}

Mechanical actuators the operating principle of which is based on the deformation of a shape-memory alloy can create high forces while the overall actuator size is comparably small. Often these kinds of actuators are operated in an on-off mode, only. Nonetheless, a continuous operation is also possible, which is usually controlled using heuristic linear or nonlinear controllers. In this article a flatness-based open-loop control of this kind of actuators is proposed using a simple physically inspired model of the alloy.

The operating principle of shape-memory alloy actuators is the diffusionless transition between different metallic phases. Depending on the phase, the geometric shape of the actuator changes thus leading to relatively large deformations. What metallic phase the alloy prefers depends on the current temperature and the mechanical stress (there are magnetic alloys, too, but these are not considered here). For a nickel titanium alloy (NiTi), there is an austenitic phase, which is stable at high temperatures, and a martensitic phase, stable at low temperatures. Because of the shape of the crystal cells, martensite forms in twins of different orientation (Wang and Sehitoglu (2014)), when the alloy is cooled. Stretching the cold alloy results in a shift of the twin plane, which looks like a plastic deformation from the macroscopic point of view, but is a diffusionless process. The process can be reversed by heating the alloy, which turns the crystal in the austenitic phase again, so that the previous shape results.

To this end, actuators formed as thin wires are considered, which can only afford tension forces in one direction. Thus, the martensite cells will orientate in a way that minimizes the strain energy and different orientations are not subject to the considerations, here. This way, one can regard the phase fractions $x_{\mathrm{A}}$ of austenite and the fractions $x_{+}$and $x_{-}$of representative martensite phases, only (Müller and Seelecke (2001)). In addition, the following simplified assumptions are made: The wire is always loaded by a tension force $f(l, i, \ldots)$ that can be expressed by the wire length $l$ and its derivatives. This function is given by the mechanical system attached

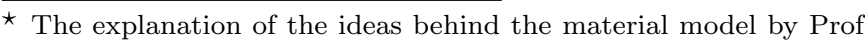
Seelecke is gratefully acknowledged.
}

to the wire. The mass, and therefore all inertial forces, of the wire can be neglected. The wire shall have a homogeneous temperature, spatially distributed effects are not considered yet. Thus, a wire of spatially constant cross section $A$, volume $V$, and length $l=l_{0}(1+\epsilon)$ is considered, where $l_{0}$ is the length in the austenitic phase under no load and $\epsilon$ is the strain. Note that the cross section $A$ depends on both the phase fraction and the strain, because of the volume preserving phase transition and the elastic tension of the compressible material, while the volume depends on the elastic component of the strain only. Polycrystalline effects are neglected and the wire is described by stacked layers of martensite and austenite with the fractions $x_{+}=x$ and $x_{\mathrm{A}}=1-x$, respectively. Since the wire is assumed to be always loaded, only one martensitic phase is considered.

\section{MATERIAL MODEL}

To explain the hysteretic behavior of the shape-memory alloy, a free energy model was developed in Müller and Seelecke (2001), which has been used in many other works. In this model, the (Helmholtz) free energy $F=$ $U-T S$ as a function of the temperature $T$ and the strain $\epsilon$ is considered. Here, $U$ denotes the internal energy and $S$ the entropy. A thermodynamic system tends to minimize the free energy. Considering the material tension with respect to austenite, the martensitic phases can be regarded to have a strain $\epsilon_{ \pm}$, so that the (linear) stressstrain relation is $\sigma_{\mathrm{A}}=E_{\mathrm{A}} \epsilon$ and $\sigma_{ \pm}=E_{ \pm}\left(\epsilon-\epsilon_{ \pm}\right)$for austenite and martensite, respectively, while $E$ denotes the corresponding Young's modulus. Therefore, the free energy has minima at the specific strains, if the phases are stable at the temperature $T$. At high temperatures, the entropy term dominates and only the austenitic phase is stable (see Figure 1a)). As the temperature decreases, minima at the martensitic phases occur, but the energy barrier prevents a phase transition (Figure 1b)). If the temperature decreases further, the energy barrier gets smaller so that the alloy transforms to the martensitic phase (Figure 1c)). As the material is heated again, there is an energy barrier to the austenite phase, which explains the hysteresis.

For an alloy under tension stress $\sigma$, the free enthalpy $G=F-\partial_{\epsilon} U \epsilon$ is minimized. To find the term $\partial_{\epsilon} U$, the Gibbs fundamental equation $\mathrm{d} U=T \mathrm{~d} S+\mathrm{d} W$ conforming to Kluge and Neugebauer (1994) with the work term 


$$
\mathrm{d} W=f \mathrm{~d} l=\sigma A l_{0} \mathrm{~d} \epsilon=\sigma \frac{V}{1+\epsilon} \mathrm{d} \epsilon
$$

in combination with the total derivative $\mathrm{d} U=\partial_{S} U \mathrm{~d} S+$ $\partial_{\epsilon} U \mathrm{~d} \epsilon$ can be used to find

$$
\partial_{\epsilon} U=\sigma \frac{V}{1+\epsilon}
$$

The additional term $\partial_{\epsilon} U \epsilon$ in the free enthalpy explains why only one martensitic phase is formed when cooling a wire under tension.

This phase transformation process is assumed to have a rate that depends on the temperature and the height $\Delta G(\sigma, T)$ of the energy barriers, which are different for the different transitions $\mathrm{A}+$ from austenite to martensite and + A from martensite to austenite. There, the quantities $T$ and $\Delta G$ occur in the argument of an exponential function. For an explanation the reader is referred to Müller and Seelecke (2001). Thus, one has quasilinear differential equations

$$
\begin{aligned}
\tau \dot{x}_{\mathrm{A}} & =x_{+} p_{+\mathrm{A}}(T, \sigma)-x_{\mathrm{A}} p_{\mathrm{A}+}(T, \sigma) \\
\tau \dot{x}_{+} & =x_{\mathrm{A}} p_{\mathrm{A}+}(T, \sigma)-x_{+} p_{\mathrm{A}+}(T, \sigma)
\end{aligned}
$$

for the phase fractions, which obviously respect the algebraic condition $x_{\mathrm{A}}+x_{+}=1$ and can be reduced to

$$
\tau \dot{x}=(1-x) p_{\mathrm{A}+}(\sigma, T)-x p_{+\mathrm{A}}(\sigma, T) .
$$

Since the proposed time constant $\tau$ is very small, singular perturbation theory may be used to come up with algebraic equations instead, neglecting the very fast dynamics.

To complete the actuator model, an equation for the heating power $P$ is required. The following considerations are easier to understand when working with the length $l$ and force $f$ of the wire. Since $\dot{U}=\dot{Q}+f i$ for the change of the internal energy, for the enthalpy $H=U-f l$ one has $\dot{H}=\dot{Q}-l \dot{f}$. On the other hand $H=x H_{\mathrm{M}}+(1-x) H_{\mathrm{A}}$ holds for the enthalpy, where $H_{\mathrm{M}}$ and $H_{\mathrm{A}}$ is the enthalpy of the whole wire, as if it had only one metallic phase. Further

$$
\dot{H}_{\mathrm{M}, \mathrm{A}}=\partial_{T} H_{\mathrm{M}, \mathrm{A}} \dot{T}+\partial_{f} H_{\mathrm{M}, \mathrm{A}} \dot{f},
$$

while $\partial_{f} H_{\mathrm{M}, \mathrm{A}}=-l$. Comparing the two equations for the derivative $\dot{H}$, one finds

$$
\begin{aligned}
\dot{H} & =\dot{x}\left(H_{\mathrm{M}}-H_{\mathrm{A}}\right)-l \dot{f}+\left(x \partial_{T} H_{\mathrm{M}}+(1-x) \partial_{T} H_{\mathrm{A}}\right) \dot{T} \\
& =\dot{Q}-l \dot{f},
\end{aligned}
$$

which yields

$$
\dot{Q}=\left(x \partial_{T} H_{\mathrm{M}}+(1-x) \partial_{T} H_{\mathrm{A}}\right) \dot{T}+\left(H_{\mathrm{M}}-H_{\mathrm{A}}\right) \dot{x} .
$$

\section{DIFFERENTIALLY FLAT SYSTEM}

Combining the shape-memory alloy wire model with the mechanically system providing an external force (a spring for instance) results in a differentially flat system with the wire length $l$ as a flat output, as will be shown next. Given the length $l_{0}$ of the unloaded wire in austenitic phase as a parameter, one has the relation $l=l_{0}(1+\epsilon)$ and that knowing $l$ the strain is known, too. From the mechanical system itself one directly computes the tension force $f=f(l, i, \ldots)$.

To compute the stress $\sigma=\frac{f}{A}$ and the phase fractions the cross section $A$ of the wire is required first. Under the assumptions above, one has a volume preserving tension due to the phase transition next to an elastic tension due to stress. The latter effect contributes a strain

$$
\bar{\epsilon}=\sigma\left(\frac{1-x}{E_{\mathrm{A}}}+\frac{x}{E_{\mathrm{M}}}\right)
$$

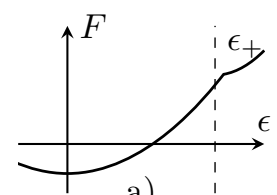

a)

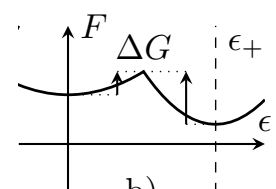

b)

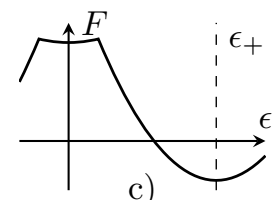

c)
Fig. 1. Free energy $F(T, \epsilon)$. Sections are shown for different temperatures $T$.

so that $\epsilon=x \epsilon_{+}+\bar{\epsilon}$ for the composite strain. The elastic tension scales the cross section by $(1-\nu \bar{\epsilon})^{2} \approx 1-2 \nu \bar{\epsilon}$ with the transverse deformation constant $\nu$ so that

$$
A=A_{0} \frac{(1-\nu \bar{\epsilon})^{2}}{1+x \epsilon_{+}}
$$

for the cross section. These equations can be solved for the phase fraction $x$ and the stress $\sigma$, given the strain $\epsilon$ and the force $f$.

Now, in the rate equation (1) the quantities computed so far as well as the derivative $\dot{x}$ of the phase fraction are substituted to solve this equation for the temperature $T$. Using (2) and differentiating $T$ then allows to express the control input, viz the heating power $P=\dot{Q}$. This way, given any sufficiently smooth trajectory $t \mapsto l(t)$, allows one to compute the corresponding control input $P(t)$.

The step of computing the temperature requires some attention. The monocrystalline model suggests that, due to the large negative exponents, there is almost no phase transition until a critical temperature or strain reached. Inverting the equations directly results in very large heating powers. In practice, such a behavior is not observed. A polycrystalline extension of this model as proposed in Heinze (2004) will allow to address this problem.

One should also note that the wire can be easily heated by forcing an electrical current through it, while there is usually no way to cool it actively. Negative values of $\dot{Q}$ are achieved by convection that depends on the temperature and geometry of the wire, which are known from $t \mapsto l(t)$.

Since the small wire is fixed at its ends, there are very large heat sinks, which call for a spatially distributed model. Such a model might lead to much better coincidence between simulation results and experiments, in return yielding more accurate control behavior.

\section{REFERENCES}

Heinze, O. (2004). A computationally efficient free energy model for shape memory alloys - experiments and theory. Ph.D. thesis, North Carolina State University.

Kluge, G. and Neugebauer, G. (1994). Grundlagen der Thermodynamik. Spektrum Akademischer Verlag, Heidelberg, Berlin, Oxford.

Müller, I. and Seelecke, S. (2001). Thermodynamic Aspects of Shape Memory Alloys. Mathematical and Computer Modelling, 34, 1307-1355.

Wang, J. and Sehitoglu, H. (2014). Martensite modulus dilemma in monoclinic NiTi-theory and experiments. International Journal of Plasticity, 61, 17-31. 